\title{
Urachal cyst revealed by pyogenic granuloma in an adult patient
}

Randa Said El Mabrouk ${ }^{1}$, Ines Lahouel ${ }^{1}$, Randa Salem ${ }^{1}$, Hichem Belhadjali ${ }^{1}$, and

Jameleddine Zili ${ }^{1}$

${ }^{1}$ Fattouma Bourguiba University Hospital of Monastir

December 14, 2021

\begin{abstract}
Urachal cyst is a rare congenital disorder due to a defect in the obliteration of the urachal duct, usually diagnosed in the first months of life. We report a case of urachal cyst revealed by a Pyogenic granuloma in an adult patient.
\end{abstract}

Title: Urachal cyst revealed by Pyogenic granuloma in an adult patient.

Running title : Persistent urachal cyst.

The category for which the article is being submitted : Clinical image

Authors:

Randa Said El Mabrouk MD (1) (2): Medical doctor of dermatology

Ines Lahouel MD (1) (2): Medical doctor of dermatology

Randa Salem MD (3): Medical doctor of radiology.

Hichem Belhadjali MDPhD (1) (2): Clinical professor of dermatology.

Jameleddine Zili MD Ph D (1) (2): Clinical professor of dermatology.

\section{Affiliations:}

(1) Dermatology department, Fattouma Bourguiba Hospital, University of Medicine, Monastir, Tunisia.

(2) Dermo-respiratory research laboratory, the University of Medicine of Monastir, Tunisia.

(3) Radiology department, University of Medicine, Monastir, Tunisia.

\section{Contributors' Statement Page:}

Randa Said El Mabrouk: Writing the manuscript and submitting the revised article.

Ines Lahouel: Writing the manuscript.

Randa Salem: Wiriting the radiology part of the manuscript.

Hichem Belhadjali: Supervised and approved the revised manuscript

Jameleddine Zili: Supervised and approved the revised manuscript

Consent statement: approved by all authors

Written informed consent was also obtained from the patient to publish this report in accordance with the journal' patient consent policy" 
Ethical statement : approved

\section{Statement of acknowledgement : none}

We choose this statement of Data availability : Data openly available in a public repository that issues datasets with DOIs

\section{Corresponding author:}

Randa Said El Mabrouk, MD,

Department of Dermatology, Fattouma Bourguiba Hospital, 5000 Monastir, Tunisia

Email: saidranda@yahoo.fr Phone: +216 54492241

Key words: Urachal cyst, pyogenic granuloma, adult.

Word counts for the text: 198

Figure number: 1

Source of support: none

\section{Acknowledgments: none}

\section{Conflict of interest: none}

\section{Case presentation :}

An 18-year-old patient consulted in dermatology for an umbilical nodule that had been evolving for 2 months. Physical examination found an umbilical pyogenic granuloma (Figure 1) with a discrete oozing and pus discharge after manual compression. An involution abnormality of the urachus duct was suspected and an abdominal ultrasound confirmed the diagnosis by showing an infected urachal cyst. The patient was treated with antibiotics and referred for a surgical removal of the cyst.

\section{Discussion:}

The urachus is a fibrous residue of the allantois that connects the bladder to the umbilicus. The involution of this structure usually occurs after birth in the form of an obliterated fibrous cord. Urachal cyst is a sub umbilical fluid collection due to the accumulation of serous secretions and blood. A urachal cyst is usually diagnosed by umbilical flow or umbilical pyogenic granuloma in newborns, and exceptionally it may remain latent and be diagnosed in adulthood. The persistence of this cyst can be revealed by complications like infection, as in our case. The exploration of the urachal cyst is essentially based on abdominopelvic ultrasound and the treatment is based on surgical excision in order to prevent the risk of infection and neoplastic degeneration.

\section{Key clinical message :}

The pathologies of the urachus, although exceptional, must be evoked when consulting for a nodule or umbilical oozing not only in children but also in adults. Early recognition of urachal cyst can decrease the risk of developing complications.

\section{Reference:}

1. Tsai M-S, Yeh M-L. Patent Urachus. N Engl J Med. 6 oct 2011;365(14):1328-1328. 


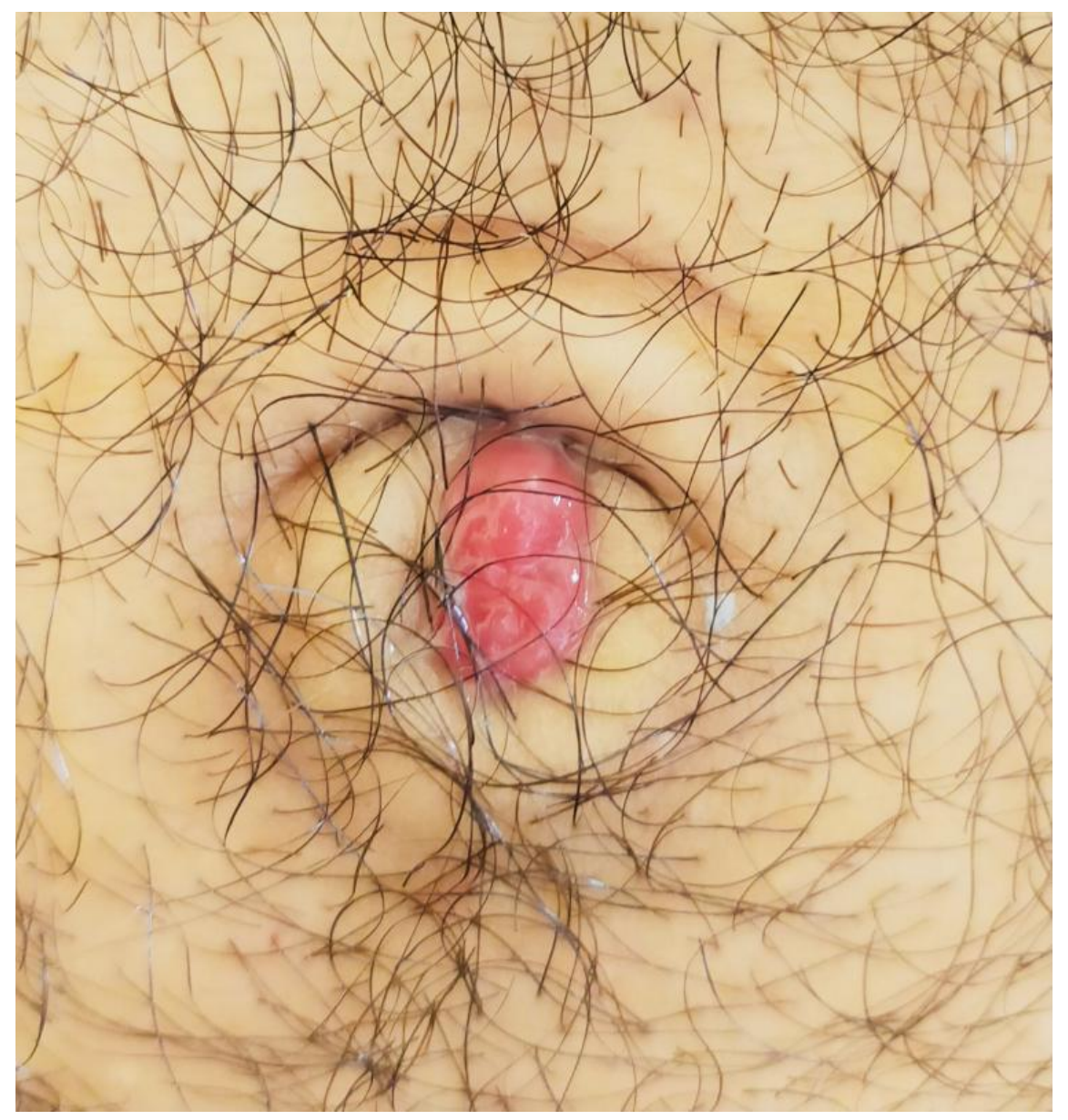

